\title{
Research Article \\ On Some Properties of New Paranormed Sequence Space of Nonabsolute Type
}

\author{
Vatan Karakaya ${ }^{1}$ and Necip Şimşek ${ }^{2}$ \\ ${ }^{1}$ Department of Mathematical Engineering, Yildiz Technical University, Davutpasa Campus, \\ Esenler 34220, Istanbul, Turkey \\ ${ }^{2}$ Department of Mathematic, Istanbul Commerce University, Üsküdar, Istanbul, Turkey
}

Correspondence should be addressed to Vatan Karakaya, vkkaya@yildiz.edu.tr

Received 25 February 2012; Accepted 24 June 2012

Academic Editor: Qiji J. Zhu

Copyright (c) 2012 V. Karakaya and N. Şimşek. This is an open access article distributed under the Creative Commons Attribution License, which permits unrestricted use, distribution, and reproduction in any medium, provided the original work is properly cited.

\begin{abstract}
We introduce some new generalized sequence space related to the space $\ell(p)$. Furthermore we investigate some topological properties as the completeness, the isomorphism, and also we give some inclusion relations between this sequence space and some of the other sequence spaces. In addition, we compute $\alpha-, \beta$-, and $\gamma$-duals of this space and characterize certain matrix transformations on this sequence space.
\end{abstract}

\section{Introduction}

In studying the sequence spaces, especially, to obtain new sequence spaces, in general, the matrix domain $\mu_{A}$ of an infinite matrix $A$ defined by $\mu_{A}=\left\{x=\left(x_{k}\right) \in w: A x \in \mu\right\}$ is used. In most cases, the new sequence space $\mu_{A}$ generated by a sequence space $\mu$ is the expansion or the contraction of the original space $\mu$. In some cases, these spaces could be overlapped. Indeed, one can easily see that the inclusion $\mu_{S} \subset \mu$ strictly holds for $\mu \in\left\{\ell_{\infty}, c, c_{0}\right\}$. Similarly one can deduce that the inclusion $\mu \subset \mu_{\Delta}$ also strictly holds for $\mu \in\left\{\ell_{\infty}, c, c_{0}\right\}$, where $S$ and $\Delta$ are matrix operators.

Recently, in [1], Mursaleen and Noman constructed new sequence spaces by using matrix domain over a normed space. They also studied some topological properties and inclusion relations of these spaces.

It is well known that paranormed spaces have more general properties than the normed spaces. In this work, we generalize the normed sequence spaces defined by Mursaleen and Noman [1] to the paranormed spaces. Furthermore we introduce new sequence space over the paranormed space. Next we investigate behaviors of this sequence 
space according to topological properties and inclusion relations. Finally we give certain matrix transformation on this sequence space and its duals.

In the literature, by using the matrix domain over the paranormed spaces, many authors have defined new sequence spaces. Some of them are as follows. For example, Choudhary and Mishra [2] have defined the sequence space $\overline{\ell(p)}$ where the $S$-transform is in $\ell(p)$, Başar and Altay [3,4] defined the spaces $\lambda(u, v ; p)=\{\lambda(p)\}_{G}$ for $\lambda \in$ $\left\{\ell_{\infty}, c, c_{0}\right\}$ and $\ell(u, v ; p)=\{\ell(p)\}_{G}$, respectively, and Altay and Başar [5] have defined the spaces $r_{\infty}^{t}(p), r_{c}^{t}(p), r_{0}^{t}(p)$. In [6], Karakaya and Polat defined and examined the spaces $e_{0}^{r}(\Delta ; p), e^{r}(\Delta ; p), e_{\infty}^{r}(\Delta ; p)$, and Karakaya et al. [7] have recently introduced and studied the spaces $\ell_{\infty}(\lambda, p), c(\lambda, p), c_{0}(\lambda, p)$, where $R^{t}$ and $E^{r}$ denote the Riesz and the Euler means, respectively, $\Delta$ denotes the band matrix of the difference operators, and $\Lambda, G$ are defined in $[1,8]$, respectively. Also, the information on matrix domain of sequence spaces can be found in $[9-13]$.

By $w$, we denote the space of all real valued sequences. Any vector subspace of $w$ is called a sequence space. By the spaces $\ell_{1}, c s$, and $b s$, we denote the spaces of all absolutely convergent series, convergent series, and bounded series, respectively.

A linear topological space $X$ over the real field $\mathbb{R}$ is said to be a paranormed space if there is a subadditivity function $h: X \rightarrow \mathbb{R}$ such that $h(\theta)=0, h(x)=h(-x)$, and scalar multiplication is continuous, that is, $\left|\alpha_{n}-\alpha\right| \rightarrow 0$ and $h\left(x_{n}-x\right) \rightarrow 0$ imply $h\left(\alpha_{n} x_{n}-\alpha x\right) \rightarrow 0$ for all $\alpha$ in $\mathbb{R}$ and $x$ in $X$, where $\theta$ is the zero in the linear space $X$.

Let $\mu, v$ be any two sequence spaces, and let $A=\left(a_{n k}\right)$ be any infinite matrix of real number $a_{n k}$, where $n, k \in \mathbb{N}$ with $\mathbb{N}=\{0,1,2, \ldots\}$. Then we say that $A$ defines a matrix mapping from $\mu$ into $v$ by writing $A: \mu \rightarrow v$, if for every sequence $x=\left(x_{k}\right) \in \mu$, the sequence $A x=\left(A_{n}(x)\right)$, the $A$-transform of $x$, is in $v$, where

$$
A_{n}(x)=\sum_{k} a_{n k} x_{k} \quad(n \in \mathbb{N})
$$

By $(\mu, v)$, we denote the class of all matrices $A$ such that $A: \mu \rightarrow v$. Thus, $A \in(\mu, v)$ if and only if the series on the right hand side of (1.1) converges for each $n \in \mathbb{N}$ and every $x \in \mu$, and we have $A x \in v$ for all $x \in \mu$. A sequence $x$ is said to be $A$-summable to $a$ if $A x$ converges to $a$ which is called as the $A$-limit of $x$.

Assume here and after that $\left(p_{k}\right),\left(q_{k}\right)$ are bounded sequences of strictly positive real numbers with sup $p_{k}=H$ and $M=\max (1, H)$, and also let $p_{k}=p_{k} /\left(p_{k}-1\right)$ for $1<p_{k}<\infty$ and for all $k \in \mathbb{N}$. The linear space $\ell(p)$ was defined by Maddox [14] as follows:

$$
\ell(p)=\left\{x=\left(x_{n}\right) \in w: \sum_{n=0}^{\infty}\left|x_{n}\right|^{p_{n}}<\infty\right\}
$$

which are the complete space paranormed by

$$
h(x)=\left(\sum_{n=0}^{\infty}\left|x_{n}\right|^{p_{n}}\right)^{1 / M} .
$$

Throughout this work, by $\digamma$ and $N_{k}$, respectively, we will denote the collection of all subsets of $\mathbb{N}$ and the set of all $n \in \mathbb{N}$ such that $n \geq k$ and $e=(1,1,1, \ldots)$. 


\section{The Sequence Space $\ell(\lambda, p)$}

In this section, we define the sequence space $\ell(\lambda, p)$ and prove that this sequence space according to its paranorm is complete paranormed linear space. In [1], Mursaleen and Noman defined the matrix $\Lambda=\left(\lambda_{n k}\right)_{n, k=0}^{\infty}$ by

$$
\lambda_{n k}= \begin{cases}\frac{\lambda_{k}-\lambda_{k-1}}{\lambda_{n}} & (0 \leq k \leq n) \\ 0 & (k>n),\end{cases}
$$

where $\lambda=\left(\lambda_{k}\right)_{k=0}^{\infty}$ is a strictly increasing sequence of positive reals tending to $\infty$, that is, $0<\lambda_{0}<\lambda_{1}<\cdots$ and $\lambda_{k} \rightarrow \infty$ as $k \rightarrow \infty$. Now, by using (2.1) we define new sequence space as follows:

$$
\ell(\lambda, p)=\left\{x=\left(x_{k}\right) \in w: \sum_{n=0}^{\infty}\left|\frac{1}{\lambda_{n}} \sum_{k=0}^{n}\left(\lambda_{k}-\lambda_{k-1}\right) x_{k}\right|^{p_{n}}<\infty\right\} .
$$

For any $x=\left(x_{n}\right) \in w$, we define the sequence $y=\left(y_{n}\right)$, which will frequently be used, as the $\Lambda$-transform of $x$, that is, $y=\Lambda(x)$, and hence

$$
y_{n}=\sum_{k=0}^{n}\left(\frac{\lambda_{k}-\lambda_{k-1}}{\lambda_{n}}\right) x_{k} \quad(n \in N)
$$

We now may begin with the following theorem.

Theorem 2.1. The sequence space $\ell(\lambda, p)$ is the complete linear metric space with respect to paranorm defined by

$$
h(x)=\left(\sum_{n=0}^{\infty}\left|\frac{1}{\lambda_{n}} \sum_{k=0}^{n}\left(\lambda_{k}-\lambda_{k-1}\right) x_{k}\right|^{p_{n}}\right)^{1 / M} .
$$

Proof. The linearity of $\ell(\lambda, p)$ with respect to the coordinatewise addition and scalar multiplication follows from the following inequalities which are satisfied for $x, t \in \ell(\lambda, p)$ (see, [15]):

$$
\begin{aligned}
\left(\sum_{n=0}^{\infty}\left|\frac{1}{\lambda_{n}} \sum_{k=0}^{n}\left(\lambda_{k}-\lambda_{k-1}\right)\left(x_{k}+t_{k}\right)\right|^{p_{n}}\right)^{1 / M} \leq & \left(\sum_{n=0}^{\infty}\left|\frac{1}{\lambda_{n}} \sum_{k=0}^{n}\left(\lambda_{k}-\lambda_{k-1}\right) x_{k}\right|^{p_{n}}\right)^{1 / M} \\
& +\left(\sum_{n=0}^{\infty}\left|\frac{1}{\lambda_{n}} \sum_{k=0}^{n}\left(\lambda_{k}-\lambda_{k-1}\right) t_{k}\right|^{p_{n}}\right)^{1 / M}
\end{aligned}
$$

and for any $\alpha \in \mathbb{R}$ (see, [16])

$$
|\alpha|^{p_{k}} \leq \max \left\{1,|\alpha|^{M}\right\}
$$


It is clear that $h(\theta)=0, h(x)=h(-x)$ for all $x \in \ell(\lambda, p)$. Again inequalities (2.5) and (2.6) yield the subadditivity of $h$ and hence $h(\alpha x) \leq \max \left\{1,|\alpha|^{M}\right\} h(x)$. Let $\left\{x^{m}\right\}$ be any sequence of points $x^{m} \in \ell(\lambda, p)$ such that $h\left(x^{m}-x\right) \rightarrow 0$ and $\left(\alpha_{m}\right)$ also any sequence of scalars such that $\alpha_{m} \rightarrow \alpha$. Then, since the inequality

$$
h\left(x^{m}\right) \leq h(x)+h\left(x^{m}-x\right)
$$

holds by subadditivity of $h$, we can write that $\left\{h\left(x^{m}\right)\right\}$ is bounded and we thus have

$$
\begin{aligned}
h\left(\alpha_{m} x^{m}-\alpha x\right) & =\left(\sum_{n=0}^{\infty}\left|\frac{1}{\lambda_{n}} \sum_{k=0}^{n}\left(\lambda_{k}-\lambda_{k-1}\right)\left(\alpha_{m} x_{k}^{m}-\alpha x_{k}\right)\right|^{p_{n}}\right)^{1 / M} \\
& \leq\left|\alpha_{m} \longrightarrow \alpha\right|^{1 / M} h\left(x^{m}\right)+|\alpha|^{1 / M} h\left(x^{m}-x\right)
\end{aligned}
$$

which tends to zero as $n \rightarrow \infty$. Therefore, the scalar multiplication is continuous. Hence $h$ is a paranorm on the space $\ell(\lambda, p)$. It remains to prove the completeness of the space $\ell(\lambda, p)$. Let $\left\{x^{j}\right\}$ be any Cauchy sequence in the space $\ell(\lambda, p)$, where $x^{j}=\left\{x_{0}^{(j)}, x_{1}^{(j)}, x_{2}^{(j)}, \ldots\right\}$. Then, for a given $\varepsilon>0$, there exists a positive integer $m_{0}(\varepsilon)$ such that $h\left(x^{j}-x^{i}\right)<\varepsilon / 2$ for all $i, j>m_{0}(\varepsilon)$. Using definition of $h$, we obtain for each fixed $n \in \mathbb{N}$ that

$$
\left|\Lambda_{n}\left(x^{j}\right)-\Lambda_{n}\left(x^{i}\right)\right| \leq\left(\sum_{n=0}^{\infty}\left|\Lambda_{n}\left(x^{j}\right)-\Lambda_{n}\left(x^{i}\right)\right|^{p_{n}}\right)^{1 / M}<\frac{\varepsilon}{2}
$$

for every $i, j>m_{0}(\varepsilon)$ which leads us to the fact that $\left\{\Lambda_{n}\left(x^{0}\right), \Lambda_{n}\left(x^{1}\right), \Lambda_{n}\left(x^{2}\right), \ldots\right\}$ is a Cauchy sequence of real numbers for every fixed $n \in \mathbb{N}$. Since $\mathbb{R}$ is complete, it converges, say $\Lambda_{n}\left(x^{i}\right)-\Lambda_{n}(x)$ as $i \rightarrow \infty$. Using these infinitely many limits, we may write the sequence $\left\{\Lambda_{0}(x), \Lambda_{1}(x), \Lambda_{2}(x), \ldots\right\}$. From (2.9) as $i \rightarrow \infty$, we have

$$
\left|\Lambda_{n}\left(x^{j}\right)-\Lambda_{n}(x)\right|<\frac{\varepsilon}{2}, \quad\left(j \geq m_{0}(\varepsilon)\right)
$$

for every fixed $n \in \mathbb{N}$. By using (2.9) and boundedness of Cauchy sequence, we have

$$
\left(\sum_{n=0}^{\infty}\left|\Lambda_{n}(x)\right|^{p_{n}}\right)^{1 / M} \leq\left(\sum_{n=0}^{\infty}\left|\Lambda_{n}\left(x^{j}\right)-\Lambda_{n}\left(x^{i}\right)\right|^{p_{n}}\right)^{1 / M}+\left(\sum_{n=0}^{\infty}\left|\Lambda_{n}\left(x^{j}\right)\right|^{p_{n}}\right)^{1 / M}<\infty .
$$

Hence, we get $x \in \ell(\lambda, p)$. So, the space $\ell(\lambda, p)$ is complete.

Theorem 2.2. The sequence space $\ell(\lambda, p)$ of nonabsolute type is linearly isomorphic to the space $\ell(p)$, where $0<p_{k} \leq H<\infty$.

Proof. To prove the theorem, we would show the existence of linear bijection between the spaces $\ell(\lambda, p)$ and $\ell(p)$. With the notation of (2.3), we define transformation $T$ from $\ell(\lambda, p)$ to $\ell(p)$ by $x \rightarrow y=T x$. The linearity of $T$ is trivial. Furthermore, it is obvious that $x=\theta$ whenever $T x=\theta$ and hence $T$ is injective. 
Let $y \in \ell(p)$ and define the sequence $x=\left\{x_{n}\right\}$

$$
x_{n}(\lambda)=\sum_{k=n-1}^{n}\left((-1)^{n-k} \frac{\lambda_{k}}{\lambda_{n}-\lambda_{n-1}}\right) y_{k} \quad(n, k \in \mathbb{N})
$$

Then, we have

$$
h_{\mathcal{\ell}(\lambda, p)}(x)=\left(\sum_{n=0}^{\infty}\left|\frac{1}{\lambda_{n}} \sum_{k=0}^{n}\left(\lambda_{k}-\lambda_{k-1}\right) x_{k}\right|^{p_{n}}\right)^{1 / M}=\left(\sum_{n=0}^{\infty}\left|y_{n}\right|^{p_{n}}\right)^{1 / M}=h_{\mathcal{\ell}(p)}(y) .
$$

Thus, we have that $x \in \ell(\lambda, p)$ and consequently $T$ is surjective. Hence, $T$ is a linear bijection and this tells us that the spaces $\ell(\lambda, p)$ and $\ell(p)$ are linearly isomorphic. This completes the proof.

\section{Some Inclusion Relations}

In this section, we give some inclusion relations concerning the space $\ell(\lambda, p)$. Before giving the theorems about the section, we give a lemma given in [1].

Lemma 3.1. For any sequence $x=\left(x_{k}\right) \in w$, the equalites

$$
\begin{gathered}
S_{n}(x)=x_{n}-\Lambda_{n}(x), \\
S_{n}(x)=\frac{\lambda_{n-1}}{\lambda_{n}-\lambda_{n-1}}\left[\Lambda_{n}(x)-\Lambda_{n-1}(x)\right]
\end{gathered}
$$

hold, where the sequence $S(x)=\left\{S_{n}(x)\right\}$ is defined by

$$
S_{0}(x)=0, \quad S_{n}(x)=\frac{1}{\lambda_{n}} \sum_{k=1}^{n} \lambda_{k-1}\left(x_{k}-x_{k-1}\right) \quad(n \geq 1)
$$

Theorem 3.2. The inclusion $\ell(\lambda, p) \subset c_{0}(\lambda, p)$ holds.

Proof. Let $x \in \ell(\lambda, p)$. It can be written $\Lambda x \in \ell(p)$. By the definition of the space $\ell(p), \Lambda_{n} x \rightarrow$ $\infty$ as $n \rightarrow \infty$, we obtain $\Lambda x \in c_{0}$. Hence we get $x \in c_{0}(\lambda, p)$.

Theorem 3.3. The inclusion $\ell(\lambda, p) \subset \ell(p)$ if and only if $S(x) \in \ell(p)$ for every sequence $x \in \ell(\lambda, p)$, where $1 \leq p_{k} \leq H$.

Proof. We suppose that $\ell(\lambda, p) \subset \ell(p)$ holds and take any $x \in \ell(\lambda, p)$. Then $x \in \ell(p)$ by hypothesis. Thus we obtain from (3.1) that

$$
[h(S(x))]_{\ell(p)} \leq[h(x)]_{\ell(p)}+[h(\Lambda x)]_{\ell(p)}=[h(x)]_{\ell(p)}+[h(x)]_{\ell(\lambda, p)}
$$

which yields that $S(x) \in \ell(p)$. 
Conversely, let $x \in \ell(\lambda, p)$ be given. Then we have by the hypothesis that $S(x) \in \ell(p)$. Again by using (3.1)

$$
[h(x)]_{\ell(p)} \leq[h(S(x))]_{\ell(p)}+[h(\Lambda x)]_{\ell(p)}=[h(S(x))]_{\ell(p)}+[h(x)]_{\ell(\lambda, p)}
$$

which shows that $x \in \ell(p)$. Hence the inclusion $\ell(\lambda, p) \subset \ell(p)$ holds. This completes the proof.

\section{Theorem 3.4.}

(i) If $p_{n}>1$ for all $n \in N$, then the inclusion $\ell_{p}^{\lambda} \subset \ell(\lambda, p)$ holds.

(ii) If $p_{n}<1$ for all $n \in N$, then the inclusion $\ell(\lambda, p) \subset \ell_{p}^{\lambda}$ holds.

Proof. (i) If $p=\left(p_{n}\right)$ for all $n \in N$, then we write $\ell_{p}^{\lambda}$ in place of $\ell(\lambda, p)$. Let $x \in \ell_{p}^{\lambda}$. It is clear that $\Lambda(x) \in \ell_{p}$. One can find $m \in N$ such that $\left|\Lambda_{n}(x)\right|<1$ for all $n \geq m$. Under condition (i), we have $\left|\Lambda_{n}(x)\right|^{p_{n}}<\left|\Lambda_{n}(x)\right|$ for all $n \geq m$. Hence we get $x \in \ell(\lambda, p)$.

(ii) We suppose that $x \in \ell(\lambda, p)$. Then $\Lambda(x) \in \ell(p)$ and there exists $m \in N$ such that $\left|\Lambda_{n}(x)\right|^{p_{n}}<1$ for all $n \geq m$. To obtain the result, we consider the following inequality:

$$
\left|\Lambda_{n}(x)\right|=\left(\left|\Lambda_{n}(x)\right|^{p_{n}}\right)^{1 / p_{n}}<\left|\Lambda_{n}(x)\right|^{p_{n}}
$$

for all $n \geq m$. So, we get $x \in \ell_{p}^{\lambda}$.

\section{Some Matrix Transformations and Duals of the Space $\ell(\lambda, p)$}

In this section, we give the theorems determining the $\alpha_{-}^{-}, \beta-$, and $\gamma$-duals of the space $\ell(\lambda, p)$. In proving the theorem, we apply the technique used in [3]. Also we give some matrix transformations from the space $\ell(\lambda, p)$ into paranormed spaces $\ell(q)$ by using the matrix given in [1].

For the sequence space $\mu$ and $v$, the set $S(\mu, v)$ defined by

$$
S(\mu, v)=\left\{a=\left(a_{k}\right) \in w: a x \in v \forall x \in \mu\right\}
$$

is called the multiplier space of $\mu$ and $v$. The $\alpha-, \beta$-, and $\gamma$-duals of a sequence space $\mu$, which are, respectively, denoted by $\mu^{\alpha}, \mu^{\beta}$, and $\mu^{\gamma}$, are defined by

$$
\mu^{\alpha}=S\left(\mu, \ell_{1}\right), \quad \mu^{\beta}=S(\mu, c s), \quad \mu^{\gamma}=S(\mu, b s) .
$$

We may begin with the following theorem which computes the $\alpha$-dual of the space $\ell(\lambda, p)$.

Theorem 4.1. Let $K_{1}=\left\{k \in \mathbb{N}: p_{k} \leq 1\right\}$ and $K_{2}=\left\{k \in \mathbb{N}: p_{k}>1\right\}$. Define the matrix $D^{a}=\left(d_{n k}^{a}\right)$ by

$$
d_{n k}^{a}= \begin{cases}(-1)^{n-k} \frac{\lambda_{k}}{\lambda_{n}-\lambda_{n-1}} a_{n}, & (n-1 \leq k \leq n) \\ 0, & (0 \leq k \leq n-1) \text { or }(k>n)\end{cases}
$$


Then

$$
\begin{aligned}
& \ell_{K_{1}}^{\alpha}(\lambda, p)=\left\{a=\left(a_{n}\right) \in w: D^{a} \in\left(\ell(p) ; \ell_{\infty}\right)\right\} \\
& \ell_{K_{2}}^{\alpha}(\lambda, p)=\left\{a=\left(a_{n}\right) \in w: D^{a} \in\left(\ell(p) ; \ell_{1}\right)\right\} .
\end{aligned}
$$

Proof. We consider the following equality:

$$
a_{n} x_{n}=\sum_{k=n-1}^{n} d_{n k}^{a} y_{k}=\left(D^{a} y\right)_{n} \quad(n \in \mathbb{N})
$$

where $D^{a}=\left(d_{n k}^{a}\right)$ is defined by (4.3).

From (4.5), it can be obtained that $a x=\left(a_{n} x_{n}\right) \in \ell_{1}$ or $a x=\left(a_{n} x_{n}\right) \in \ell_{\infty}$ whenever $x \in \ell(\lambda, p)$ if and only if $D^{a} y \in \ell_{1}$ or $D^{a} y \in \ell_{\infty}$ whenever $y \in \ell(p)$. This means $a \in \ell_{K_{1}}^{\alpha}(\lambda, p)$ or $a \in \ell_{K_{2}}^{\alpha}(\lambda, p)$ if and only if $D^{a} \in\left(\ell(p) ; \ell_{1}\right)$ or $D^{a} \in\left(\ell(p) ; \ell_{\infty}\right)$. Hence this completes the proof.

The result of the Theorem above corresponds the Theorem 5.1(0, 8, 12) given in [17]. As a direct consequence of Theorem 4.1, we have the following.

Corollary 4.2. Let $K^{*}=\{k \in \mathbb{N}: n-1 \leq k \leq n\} \cap K$ for $K \in \digamma$. Then

(i) $\ell_{K_{1}}^{\alpha}(\lambda, p)=\left\{a=\left(a_{n}\right) \in w: \sup _{N} \sup _{k \in \mathbb{N}}\left|\sum_{n \in K^{*}} d_{n k}^{a}\right|^{p_{k}}<\infty\right\}$,

(ii) $\ell_{K_{2}}^{\alpha}(\lambda, p)=\bigcup_{M>1}\left\{a=\left(a_{n}\right) \in w: \sup _{K \in \digamma} \sum_{k}\left|\sum_{n \in K^{*}} d_{n k}^{a} M^{-1}\right|^{p_{k}^{\prime}}<\infty\right\}$

In the following theorem, we characterize the $\beta$ - and $\gamma$-duals of the space $\ell(\lambda, p)$.

Theorem 4.3. Let $K_{1}=\left\{k \in \mathbb{N}: p_{k} \leq 1\right\}, K_{2}=\left\{k \in \mathbb{N}: p_{k}>1\right\}$, and let $\Delta x_{k}=x_{k}-x_{k+1}$. Define the sequence $s^{1}=\left(s_{k}^{1}\right), s^{2}=\left(s_{k}^{2}\right)$ and the matrix $B^{a}=\left(b_{n k}^{a}\right)$ by

$$
\begin{gathered}
s_{k}^{1}=\Delta\left(\frac{a_{k}}{\lambda_{k}-\lambda_{k-1}}\right) \lambda_{k}, \quad s_{k}^{2}=\frac{a_{k} \lambda_{k}}{\lambda_{k}-\lambda_{k-1}} \\
b_{n k}^{a}= \begin{cases}s_{k^{\prime}}^{1} & (0 \leq k \leq n-1) \\
s_{k^{\prime}}^{2} & (k=n) \\
0, & (k>n)\end{cases}
\end{gathered}
$$

for all $n, k \in \mathbb{N}$. Then

$$
\begin{aligned}
& \ell_{K_{1}}^{\beta}(\lambda, p)=\ell_{K_{1}}^{\gamma}(\lambda, p)=\left\{a=\left(a_{n}\right) \in w: B^{a} \in\left(\ell(p) ; \ell_{\infty}\right)\right\}, \\
& \ell_{K_{2}}^{\beta}(\lambda, p)=\ell_{K_{2}}^{\gamma}(\lambda, p)=\left\{a=\left(a_{n}\right) \in w: B^{a} \in(\ell(p) ; c)\right\} .
\end{aligned}
$$


Proof. Consider the equality

$$
\sum_{k=0}^{n} a_{k} x_{k}=\sum_{k=0}^{n-1} s_{k}^{1} y_{k}+s_{n}^{2} y n=\left(B^{a} y\right)_{n} .
$$

From (4.9), it can be obtained that $a x=\left(a_{n} x_{n}\right) \in c S$ or $b s$ whenever $x=\left(x_{n}\right) \in \ell(\lambda, p)$ if and only if $B^{a} y \in c$ or $\ell_{\infty}$ whenever $y=\left(y_{k}\right) \in \ell(p)$. This means that $a=\left(a_{n}\right) \in$ $\left\{\ell_{K_{1}}^{\beta}(\lambda, p)\right.$ or $\left.\ell_{K_{2}}^{\beta}(\lambda, p)\right\}$ or $a=\left(a_{n}\right) \in\left\{\ell_{K_{1}}^{\gamma}(\lambda, p)\right.$ or $\left.\ell_{K_{2}}^{\gamma}(\lambda, p)\right\}$ if and only if $B^{a} \in(\ell(p) ; c)$ or $B^{a} \in\left(\ell(p) ; \ell_{\infty}\right)$. Hence this completes the proof.

We can write the following corollary from Theorem 4.3.

Corollary 4.4. Let $\dot{p}_{k}=p_{k} /\left(p_{k}-1\right)$ for $1<p_{k}<\infty$ and for all $k \in \mathbb{N}$. Then

(i) $\ell_{K_{1}}^{\beta}(\lambda, p)=\ell_{K_{1}}^{\gamma}(\lambda, p)=\left\{a=\left(a_{n}\right) \in w: s^{1}, s^{2} \in \ell_{\infty}(p)\right\}$,

(ii) $\ell_{K_{2}}^{\beta}(\lambda, p)=\ell_{K_{2}}^{\gamma}(\lambda, p)=\bigcup_{M>1}\left\{a=\left(a_{n}\right) \in w: s^{1} M^{-1}, s^{2} M^{-1} \in \ell\left(p^{\prime}\right) \cap \ell_{\infty}\left(p^{\prime}\right)\right\}$.

After this step, we can give our theorems on the characterization of some matrix classes concerning the sequence space $\ell(\lambda, p)$.

Let $x, y \in w$ be connected by the relation $y=\Lambda(x)$. For an infinite matrix $A=\left(a_{n k}\right)$, we have by using (4.9) of Theorem 4.3 that

$$
\sum_{k=0}^{m} a_{n k} x_{k}=\sum_{k=0}^{m-1} \tilde{a}_{n k} y_{k}+\frac{\lambda_{m}}{\lambda_{m}-\lambda_{m-1}} a_{n m} y_{m} \quad(m, n \in \mathbb{N})
$$

where

$$
\tilde{a}_{n k}=\left(\frac{a_{n k}}{\lambda_{k}-\lambda_{k-1}}-\frac{a_{n, k+1}}{\lambda_{k+1}-\lambda_{k}}\right) \lambda_{k} \quad(n, k \in \mathbb{N})
$$

The necessary and sufficient conditions characterizing the matrix mapping of the sequence space $\ell(p)$ of Maddox have been determined by Grosse-Erdmann [17]. Let $L$ and $M$ be the natural numbers and define the sets by $K_{1}=\left\{k \in \mathbb{N}: p_{k} \leq 1\right\}$ and $K_{2}=\left\{k \in \mathbb{N}: p_{k}>1\right\}$ and also let us put $\hat{p}_{k}=p_{k} /\left(p_{k}-1\right)$ for $1<p_{k}<\infty$ and for all $k \in \mathbb{N}$. Before giving the theorems, 
let us suppose that $\left(q_{n}\right)$ is a nondecreasing bounded sequence of positive real numbers and consider the following conditions:

$$
\begin{aligned}
& \sup _{N} \sup _{k \in K_{1}}\left|\sum_{n \in N} \tilde{a}_{n k}\right|^{q_{n}}<\infty, \\
& \exists M \sup _{N} \sum_{k \in K_{2}}\left|n \in N \tilde{a}_{n k} M^{-1}\right|^{p_{k}}<\infty, \\
& \exists M \sup _{k} \sum_{n}\left|\tilde{a}_{k} M^{-1 / p_{k}}\right|^{q_{n}}<\infty, \\
& \lim _{n}\left|\tilde{a}_{n k}\right|^{q_{n}}=0 \quad(\forall k \in \mathbb{N}), \\
& \forall L, \sup _{n} \sup _{k \in K_{1}}\left|\tilde{a}_{n k} L^{1 / q_{n}}\right|^{p_{k}}<\infty \text {, } \\
& \forall L, \exists M \sup _{n} \sum_{k \in K_{2}}\left|\tilde{a}_{k} L^{1 / q_{n}} M^{-1}\right|^{p_{k}}<\infty, \\
& \sup _{n} \sup _{k \in K_{1}}\left|\tilde{a}_{n k}\right|^{p_{k}}<\infty \text {, } \\
& \exists M \sup _{n} \sum_{k \in K_{2}}\left|\tilde{a}_{k} M^{-1}\right|^{p_{k}}<\infty \text {, } \\
& \forall L, \sup _{n} \sup _{k \in K_{1}}\left(\left|\tilde{a}_{n k}-\tilde{a}_{k}\right| L^{1 / q_{n}}\right)^{p_{k}}<\infty \text {, } \\
& \lim _{n}\left|\tilde{a}_{n k}-\tilde{a}_{k}\right|^{q_{n}}=0, \quad \forall k . \\
& \forall L, \exists M \sup _{n} \sum_{k \in K_{2}}\left(\left|\tilde{a}_{n k}-\tilde{a}_{k}\right| L^{1 / q_{n}} M^{-1}\right)^{p_{k}}, \\
& \exists L, \sup _{n} \sup _{k \in K_{1}}\left|\tilde{a}_{n k} L^{-1 / q_{n}}\right|^{p_{k}}<\infty \text {, } \\
& \exists L, \sup _{n} \sum_{k \in K_{2}}\left|\tilde{a}_{n k} L^{-1 / q_{n}}\right|^{p_{k}}<\infty, \\
& \left(\frac{\lambda_{k}}{\lambda_{k}-\lambda_{k-1}} a_{n k}\right)_{k=0}^{\infty} \in c_{0}(q) \quad(\forall n \in \mathbb{N}) \\
& \left(\frac{\lambda_{k}}{\lambda_{k}-\lambda_{k-1}} a_{n k}\right)_{k=0}^{\infty} \in c(q) \quad(\forall n \in \mathbb{N}) \\
& \left(\frac{\lambda_{k}}{\lambda_{k}-\lambda_{k-1}} a_{n k}\right)_{k=0}^{\infty} \in \ell_{\infty}(q) \quad(\forall n \in \mathbb{N}) .
\end{aligned}
$$

By using (4.7), (4.10), and Corollary 4.4, we have the following results. 
Theorem 4.5. One has the following:

(i) $A \in(\ell(\lambda, p): \ell(q))$ if and only if (4.12), (4.13), (4.14), and (4.25) hold,

(ii) $A \in\left(\ell(\lambda, p): c_{0}(q)\right)$ if and only if (4.15), (4.16), (4.17), and (4.25) hold,

(iii) $A \in(\ell(\lambda, p): c(q))$ if and only if (4.18), (4.19), (4.20), (4.21), (4.22), and (4.26) hold,

(iv) $A \in\left(\ell(\lambda, p): \ell_{\infty}(q)\right)$ if and only if (4.23), (4.24), and (4.27) hold.

\section{References}

[1] M. Mursaleen and A. K. Noman, "On the spaces of $\lambda$-convergent and bounded sequences," Thai Journal of Mathematics, vol. 8, no. 2, pp. 311-329, 2010.

[2] B. Choudhary and S. K. Mishra, "On Köthe-Toeplitz duals of certain sequence spaces and their matrix transformations," Indian Journal of Pure and Applied Mathematics, vol. 24, no. 5, pp. 291-301, 1993.

[3] F. Başar and B. Altay, "Some paranormed sequence spaces of non-absolute type derived by weighted mean," Journal of Mathematical Analysis and Applications, vol. 319, no. 2, pp. 494-508, 2006.

[4] B. Altay and F. Başar, "Generalization of the sequence space $\ell(p)$ derived by weighted mean," Journal of Mathematical Analysis and Applications, vol. 330, no. 1, pp. 174-185, 2007.

[5] B. Altay and F. Başar, "On the paranormed Riesz sequence spaces of non-absolute type," Southeast Asian Bulletin of Mathematics, vol. 26, no. 5, pp. 701-715, 2003.

[6] V. Karakaya and H. Polat, "Some new paranormed sequence spaces defined by Euler and difference operators," Acta Universitatis Szegediensis, vol. 76, no. 1-2, pp. 87-100, 2010.

[7] V. Karakaya, A. K. Noman, and H. Polat, "On paranormed $\lambda$-sequence spaces of non-absolute type," Mathematical and Computer Modelling, vol. 54, no. 5-6, pp. 1473-1480, 2011.

[8] E. Malkowsky and E. Savas, "Matrix transformations between sequence spaces of generalized weighted means," Applied Mathematics and Computation, vol. 147, no. 2, pp. 333-345, 2004.

[9] B. Altay, F. Başar, and M. Mursaleen, "On the Euler sequence spaces which include the spaces $\ell_{p}$ and $\ell_{\infty} . I_{, \prime}$ Information Sciences, vol. 176, no. 10, pp. 1450-1462, 2006.

[10] F. Başar and B. Altay, "On the space of sequences of $p$-bounded variation and related matrix mappings," Ukrainian Mathematical Journal, vol. 55, no. 1, pp. 136-147, 2003.

[11] M. Mursaleen and A. K. Noman, "On some new sequence spaces of non-absolute type related to the spaces $\ell_{P}$ and $\ell_{\infty}$ I," Filomat, vol. 25, no. 2, pp. 33-51, 2011.

[12] M. Mursaleen and A. K. Noman, "On some new sequence spaces of non-absolute type related to the spaces $\ell_{P}$ and $\ell_{\infty}$ II," Mathematical Communications, vol. 16, no. 2, pp. 383-398, 2011.

[13] M. Mursaleen, F. Başar, and B. Altay, "On the Euler sequence spaces which include the spaces $\ell_{p}$ and $\ell_{\infty}$. II," Nonlinear Analysis, vol. 65, no. 3, pp. 707-717, 2006.

[14] I. J. Maddox, "Spaces of strongly summable sequences," The Quarterly Journal of Mathematics, vol. 18, no. 2, pp. 345-355, 1967.

[15] I. J. Maddox, Elements of Functional Analysis, Cambridge University Press, Cambridge, UK, 2nd edition, 1988.

[16] I. J. Maddox, "Paranormed sequence spaces generated by infinite matrices," Mathematical Proceedings of the Cambridge Philosophical Society, vol. 64, pp. 335-340, 1968.

[17] K.-G. Grosse-Erdmann, "Matrix transformations between the sequence spaces of Maddox," Journal of Mathematical Analysis and Applications, vol. 180, no. 1, pp. 223-238, 1993. 


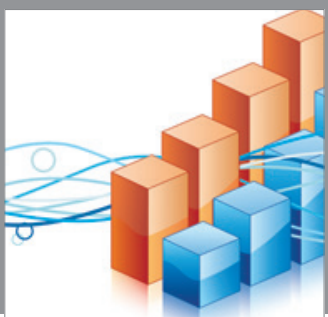

Advances in

Operations Research

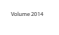

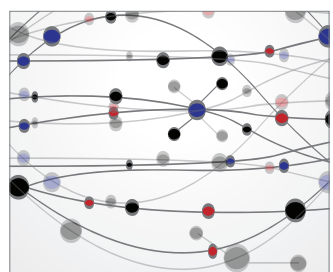

\section{The Scientific} World Journal
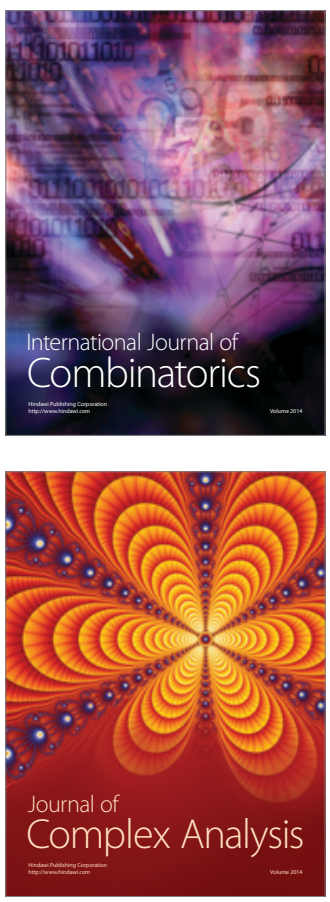

International Journal of

Mathematics and

Mathematical

Sciences
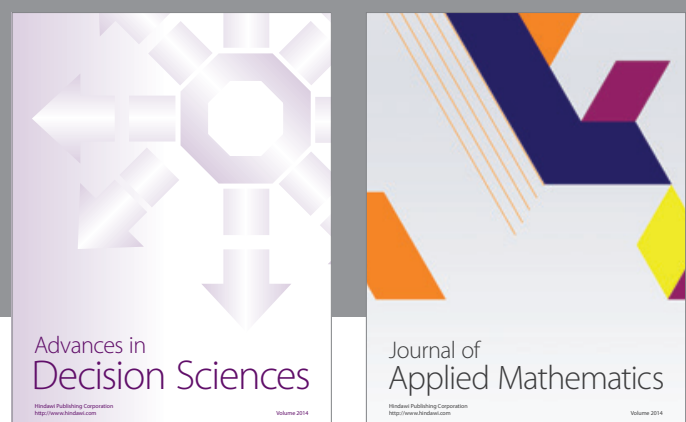

Journal of

Applied Mathematics
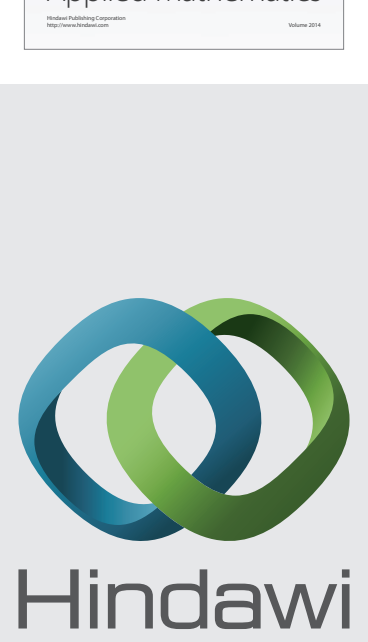

Submit your manuscripts at http://www.hindawi.com
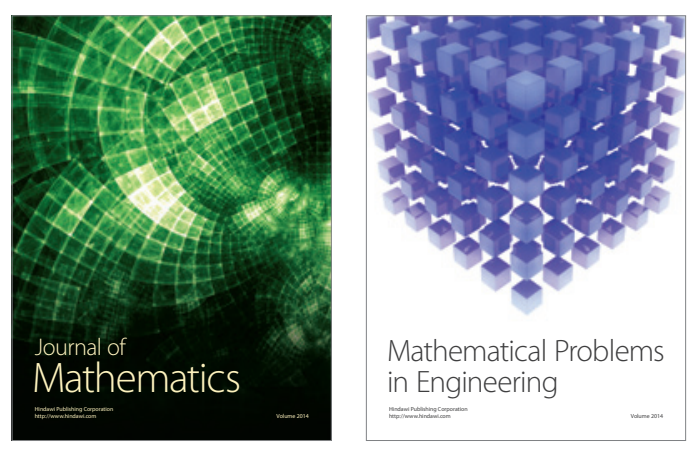

Mathematical Problems in Engineering
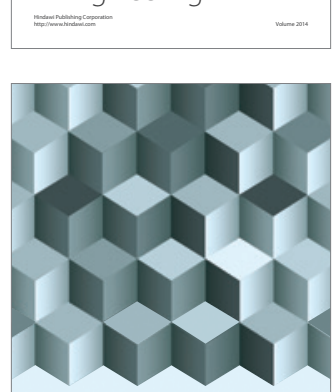

Journal of

Function Spaces
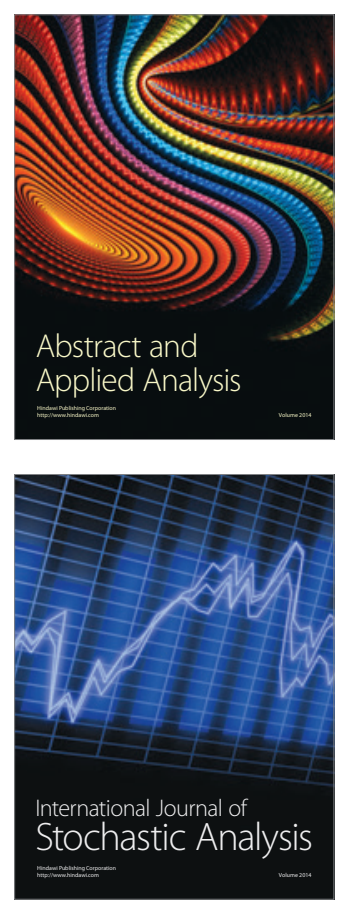

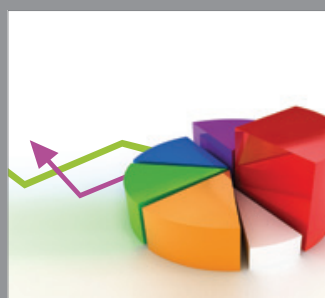

ournal of

Probability and Statistics

Promensencen
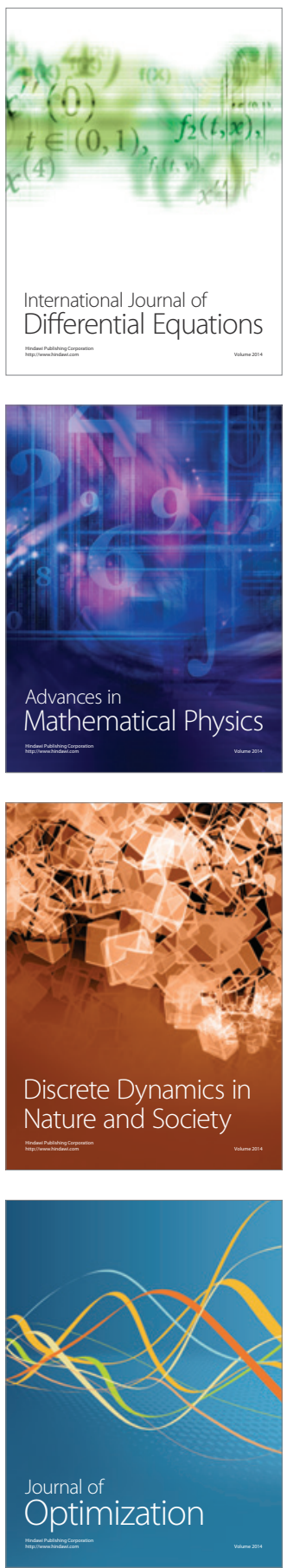